\title{
E1 feminismo en debate: mortalidad, maternidad y puericultura. Diálogo del discurso feminista con discursos sociales en la década del treinta en Chile ${ }^{1}$
}

\author{
Claudia Montero \\ University of Essex \\ claudimontero@gmail.com
}

\begin{abstract}
RESUMEN: El presente artículo examina la construcción del discurso feminista y su relación con los discursos sociales del higienismo y la eugenesia durante los años treinta. Recurriendo al análisis del discurso se aborda un corpus de revistas de organizaciones feministas del período, en particular, las editadas por el Partido Cívico Femenino y el Movimiento Pro Emancipación de la Mujer Chilena. Se enfatiza la lectura de debates sobre la salud de las mujeres, explorando las operaciones de legitimación del discurso feminista, las que terminan por construir un discurso heterogéneo, con muchas voces y alternativas para relacionarse con otros discursos sociales.
\end{abstract}

Palabras Clave: feminismo, Chile, discursos sociales, eugenesia, higienismo. 


\section{Feminism on Debate: Mortality, maternity and childcare. Dialogue OF FEMINIST DISCOURSE WITH SOCIAL DISCOURSES IN CHILE DURING THE 1930s}

ABSTRACT: The present paper examines the construction of feminist discourse and its relationship with the social discourses of hygienism and eugenics during the 1930s. Drawing on the insights of discourse analysis, I approach a corpus of magazines edited by feminist organisations during the period, in particular those of the Partido Cívico Femenino and the Movimiento Pro Emancipación de la Mujer Chilena. Emphasis is placed on the debates concerning the health of women, exploring the operations that legitimise feminist discourse, and that end up building an heterogeneous discourse, with many voices and alternatives vis-à-vis other social discourses.

KeYwords: Feminism, Chile, social discourses, eugenics, hygienism.

La pregunta por la construcción del discurso feminista en América Latina ha tenido un extenso desarrollo en las últimas décadas, llegando a conclusiones como la definición de un discurso feminista específico para América Latina, y dentro de él, una diversidad de sujetos y discursos feministas. En general, se centra en la producción escrita de las feministas latinoamericanas utilizando el análisis de discurso, lo que implica analizar el texto en su contexto. Este trabajo se inscribe en esta tradición, incluyendo un elemento más, que es considerar el diálogo del discurso feminista con otros discursos sociales, buscando contestar preguntas de diverso orden, por ejemplo: ¿Cómo se ha construido a sí mismo el feminismo? ¿Cómo se relaciona con otros discursos sociales? ¿Se puede afirmar que el feminismo es un discurso limpio? ¿Hace operaciones de legitimación?

El objetivo es analizar la construcción del discurso feminista en Chile de la década del treinta examinando los debates en torno a los temas de salud que competen específicamente a las mujeres. Se busca revisar cómo se ha construido el discurso feminista en su relación dialógica con otros discursos sociales como el higienismo, la eugenesia y el discurso del Estado. A partir de un ambiente político tensado por una crisis política y económica, en la década del treinta el feminismo en Chile radicalizó sus demandas por la mejora de la condición de las mujeres. Dadas las altas tasas de mortalidad, desnutrición y pobreza, los temas de salud se 
transformaron en uno de los ejes de las demandas feministas de la época, ya que afectaban especialmente a las mujeres en relación con la mortalidad infantil, la maternidad y la puericultura. En esa década se vivió una alta participación de mujeres en organizaciones políticas, sociales y culturales. Para este trabajo se recogen los discursos emitidos por dos organizaciones feministas: el Partido Cívico Femenino (PCF) y el Movimiento Pro Emancipación de la Mujer Chilena (МEMCH) a través de sus órganos de difusión que fueron las revistas Acción Femenina (Santiago, 1934-1939) y La Mujer Nueva (Santiago, 1935-1942), respectivamente. Ambas son organizaciones con discurso y práctica feminista de gran presencia en el país y que publicaron sus revistas a lo largo de la década y con una periodicidad mensual.

\section{DISCURSO FEMINISTA Y DISCURSO SOCIAL}

Un discurso es cualquier emisión, ya sea oral, escrita o gráfica, que funciona como un todo de significación. Son desarrollos sémicos mayores, visiblemente unificados, diferenciables, y que recorren el cuerpo de un texto, el que puede ser un escrito, una imagen y/o un objeto entre múltiples posibilidades. A la vez, un texto puede ser receptáculo de más de un discurso, los que no necesariamente están en acuerdo entre sí (Rojo 23). El discurso parte con la emisión, en la que se debe considerar que el lenguaje es múltiple y da cuenta de grupos sociales. De tal forma, la emisión refleja al sujeto que la realiza, y a la vez lo refracta. A partir de aquí podemos avanzar en el análisis de discursos feministas, considerando que todos los usuarios de esa comunidad, utilizan los mismos signos relativos a los problemas de sexo-género. A la vez, ser mujer y feminista no implica necesariamente formar una comunidad sígnica específica; ya que cada signo dentro del discurso feminista estaría marcado con acentos provenientes de distintas direcciones, por lo que siempre hay que considerar al emisor (Rojo 27). Esta idea se refuerza con la noción de que en cada texto coexisten distintas corrientes discursivas, las que no necesariamente se neutralizan entre sí, en virtud de que los textos no son estructuras monológicas. Esta característica surge de las consideraciones sobre la lucha por la hegemonía, que para nuestro caso serán aplicadas a los discursos sobre sexo-géneros, que definen un orden donde lo femenino ocupa el lugar de la subordinación. 
La emisión de un discurso es un acto individual y que expresa la subjetividad. Uno de sus objetivos es la representación de sí, en función de cómo el sujeto quiere verse y cómo quiere que lo vean. Frente a otro "por el mero hecho de dirigirse a otro, el que habla de sí mismo instala al otro en sí mismo, y por lo tanto se aprehende, se confronta y se establece como él aspira a ser, y finalmente se historiza en esta historia completa o fraudulenta" (Rojo 31). La condición del diálogo es el lenguaje e implica no solo dos subjetividades enfrentadas, sino también la relación de los discursos hacia fuera; en el caso nuestro, la relación dialógica entre discursos feministas y otros discursos sociales, en la que se pueden dar relaciones de complicidad, si los discursos colaboran, de coexistencia pacífica, cuando solamente se toleran, o de contradicción, cuando hay conflicto entre ellos (Rojo 61).

La teoría de género ha cuestionado los discursos que traducen las codificaciones jerárquicas que la cultura asigna a lo masculino/femenino, estableciendo la categoría de género-sexual. Ésta se utiliza para dar cuenta de la diferencia de la experiencia vital de varones y mujeres, la misma que ha servido para producir una jerarquización social que atraviesa a los sujetos y que se expresa en sus discursos. En lo específico, los discursos que se refieren al sexo-género necesariamente se estructuran en relación con otros discursos, y lo hacen a partir de afirmaciones reactivas o contestatarias; crean cadenas de proposiciones discursivas de reproducción cultural, o contradiscursos, como es el caso del discurso feminista. Por lo tanto, todo discurso referido a cuestiones genérico-sexuales posee un discurso contrario, estableciendo un diálogo que puede ser contenido, consciente o inconsciente (Grau et al. 24).

Por otra parte, existen relaciones de sincronía e intertextualidad entre distintos discursos y campos discursivos con lo que se define el "discurso social" -que es el conjunto de la producción discursiva de una sociedad-, y está compuesto por la totalidad de los signos elaborados por ella, considerando los discursos provenientes de las diversas prácticas sociales y las formas de conocer y significar lo conocido. Con ello, cada texto o imagen, y los discursos que lo recorren, tienen sentido en un contexto histórico y social determinado, el que deja su marca en las producciones culturales, definiendo a todo objeto que esté cubierto por su entorno (Angenot 69-96). 
El análisis del discurso entrega importantes elementos para comprender la situación de las mujeres en relación con el poder discursivo. Se debe observar esta relación en momentos determinados, permitiendo que las mujeres logren un "impacto significativo en la producción y distribución de significados con perspectiva crítica de género" (Grau et al. 17). Se hace necesario que las mujeres se apropien y resignifiquen los bienes culturales, para que adquieran poder simbólico en la sociedad; de tal forma, es preciso que creen estrategias discursivas que visibilicen sus perspectivas, con la consideración de que las mujeres no buscan ni han buscado ocupar un lugar de poder, sino que su objetivo es "desarrollar lenguajes, ideas y razonamientos que sean capaces de dominar y distribuir, construyendo un aparato representacional crítico de las relaciones de género" (Grau et al. 19). Si consideramos que este análisis recoge los discursos feministas a través de revistas en los treinta, las sujetos que los emitieron tejieron estrategias en un ejercicio doble: por una parte elaboraron discursos feministas, como forma de expresión, y a la vez, se constituyeron a sí mismas como colectivo político y social.

LA CONFORMACIÓN DEL DISCURSO FEMINISTA: EL FEMINISMO COMO FENÓMENO DE LA MODERNIDAD

En términos generales, el feminismo hace referencia a un discurso que surgió de la queja de las mujeres -ya sea individual o colectiva- acerca de su condición subordinada dentro del sistema patriarcal, reivindicando una situación de mejora vital. Esta subordinación está dada por relaciones de sexo-género, que evidencian una dominación sobre las mujeres ejercida por los varones y las instituciones sociales de predominio masculino (de Miguel 217). El discurso feminista se articuló en virtud de la configuración de un movimiento social, relacionado con momentos históricos específicos, que llevó a las mujeres a plantear teórica y prácticamente ciertas reivindicaciones basadas en dos ideas fundamentales: por un lado, la lucha por la igualdad sexo-genérica en los planos político, social y económico; y por otro, la transformación profunda de la sociedad, que implica la eliminación de las jerarquías construidas sobre la base del ser varón o ser mujer (Sau 122). 
En términos estrictos, el feminismo es propio de la modernidad, su presencia como discurso social dentro del espacio público se hizo posible gracias a la coyuntura histórica de la Revolución francesa, aunque su desarrollo consistente y su catalogación con el término "feminismo" no se dio sino hasta la segunda mitad del siglo $\mathrm{XIX}^{2}$. En este sentido, si entendemos que el discurso de la modernidad es esencialmente contradictorio, que permite dentro de sí el surgimiento de un pensamiento crítico que lo alimenta y que a la vez busca superar sus limitaciones epocales (Berman 89), los feminismos emergen como contradiscursos que representan voces alternativas y que expresan imaginarios diferentes a la simbólica oficial. La complejidad del fenómeno del feminismo y su tránsito a través de la historia contemporánea da cuenta de una multiplicidad de manifestaciones de modo que debe entenderse en plural, hablando de múltiples "feminismos" (Nash). En conjunto, los discursos feministas representaron un rechazo a las tradiciones culturales de Occidente en torno a la idea de la subordinación femenina dentro de la sociedad. De tal modo que las mujeres que abrazaron el feminismo y que pensaban de distinta forma la emancipación femenina, se diferenciaron del conjunto de las mujeres que pertenecían a otro tipo de organizaciones, como la beneficencia (Anderson y Zinsser 379).

En América Latina el desarrollo del feminismo fue más tardío que en Europa y Estados Unidos, y estuvo fuertemente influenciado por esas experiencias. Las primeras manifestaciones se dieron hacia 1870, coincidentemente con los cambios provocados por la modernización. En un principio, el feminismo fue desarrollado por mujeres de elite, quienes se acercaron al feminismo liberal; luego, a medida que las tensiones sociales se agudizaron, aparecieron otros discursos feministas asociados con el desarrollo del movimiento de mujeres obreras y de clase media. La sociedad dio una respuesta hostil a los discursos de la igualdad, por la influencia del catolicismo y por las características del liberalismo latinoamericano. En este sentido, la Iglesia, echando mano al imaginario de la Virgen María, reforzó la idea de la existencia de esferas separadas para varones y

2 Mary Nash apunta que investigaciones históricas han definido la aparición del término feminismo en textos académicos o de divulgación hacia 1870. Si bien no queda del todo claro su origen, la invención de éste se le atribuye a la francesa Hubertine Auclert, fundadora de la primera sociedad francesa de sufragio femenino (63). 
mujeres, y la consecuente subordinación femenina. Además, el liberalismo que se desarrolló en Latinoamérica defendió posturas que validaron las diferencias biológicas entre los sexo-géneros, que justificaron esta misma situación (Molyneux 78-79). Para América Latina se habla de una tipología específica: el feminismo compensatorio, cuyo discurso se caracterizó por combinar la igualdad legal con la protección de las mujeres en función de su rol de madre. Este feminismo encontró su justificación en la cultura maternalista latinoamericana, puesto que consideró la maternidad como el único lugar de autoridad para las mujeres en esta cultura. Este tipo de feminismo permitió a las mujeres salvar el conflicto entre la liberación personal y la liberación de género. Sin embargo, esta definición ha sido rebatida por quienes reconocen diferentes tipologías de feminismos en Latinoamérica y el Cono Sur, que resultan de la complejización del análisis al introducir las variables de clase social y formación ideológica.

\section{ACCIÓN POLÍTICA FEMENINA Y FEMINISTA EN EL ChILE DE LOS TREINTA}

La década del treinta en Chile fue escenario de la intensificación de la acción política de las mujeres. Con una experiencia de más de tres décadas de participación, las mujeres de los distintos grupos sociales e ideológicos se hicieron notar en el escenario político y social con demandas específicas, y también con críticas frente a la crisis política y social que enfrentó el país durante esa década. Dentro de la periodización de la historia de las mujeres en Chile, la década del treinta se denomina "tiempo de políticas" (Kirkwood), momento en el que el feminismo se entroncó con el movimiento sufragista y de este modo generó una gran participación de las mujeres en el mundo público, consolidando la participación de las mujeres de clase media en la escena política.

La realidad de las mujeres cambió considerablemente en relación con las décadas anteriores y, además, entre los distintos grupos de mujeres se dieron diferencias en sus condiciones de vida. La crisis económica empujó a muchas mujeres de clase media a buscar trabajo por primera vez, debido a la cesantía de padres o esposos. La situación de las mujeres rurales estaba cruzada por el tipo de vida inquilina que las supeditaba al marido y al patrón, además de estar marginadas del proceso de modernización, sin acceso a la salud y educación. Por otra parte, se presentaban las mujeres 
cuyas familias habían migrado hacia las ciudades, en busca de trabajo, y que se instalaron en conventillos y poblaciones a las afueras de la ciudad (Antezana-Pernet 32-33). Para las mujeres de la ciudad se mantenía la inequidad en los sueldos: las obreras recibían salarios de hambre y en general existía una amplia brecha entre el sueldo de varones y mujeres por el mismo trabajo, asunto que se transformó en una de las demandas feministas más importantes de la década. Se sumaba a esta realidad la ausencia de protección en la legislación laboral, y lo poco que había, no se cumplía; como consecuencia, el trabajo asalariado no era garantía de sobrevivencia para las mujeres. Por su parte, la realidad legal de Chile, establecía que las mujeres estaban subordinadas a un hombre durante toda su vida, ya fuera el padre o el marido; por ejemplo, una mujer casada no podía trabajar sin la autorización del marido, ni administrar sus bienes, ni tener derechos legales sobre los hijos. Lo anterior se acompañaba de la alta valoración social que tenía el papel tradicional de las mujeres: estar relegada al espacio privado de lo doméstico. Estas restricciones de los derechos civiles y políticos, chocaban con las prácticas sociales y políticas que las mujeres desarrollaban desde la década anterior.

\section{El Partido Cívico Femenino y Acción Femenina}

El Partido Cívico Femenino fue uno de los primeros partidos políticos de mujeres en Chile, fundado en 1922. Durante su primera década de funcionamiento, el partido se definió feminista, pero de un feminismo moderado. Esto significaba defender la igualdad de las mujeres en el plano civil y político, a través de la consecución de sus derechos, sin atacar ideológicamente el orden social que definía la desigualdad de las mujeres. De tal forma, Kirkwood (152) define el feminismo del Partido Cívico Femenino como moderado, moralizante y con un leve atisbo de revolución sombría. Estas afirmaciones han llevado a considerar al PCF como una organización de mujeres y no de feministas. En esta lógica encontramos a autoras como Gaviola, que afirman que la organización no tenía una tendencia ideológica clara, dada su moderación, y porque sería una organización caracterizada por una extrema heterogeneidad entre sus miembros (64). Sin embargo, es necesario reafirmar la calificación de feminista para el Partido Cívico Femenino, ya que, y siguiendo a la misma 
Kirkwood, se debe considerar el momento en el que se formó el partido: uno en el que las mujeres estaban en absoluta indefensión. También debe tenerse en cuenta que estos fueron sus primeros ejercicios en la formación de organizaciones estables y con una presencia importante en el espacio público. Si bien no está falto de tensiones, su discurso muestra una crítica a la sujeción de las mujeres, y plantea una fuerte defensa de los derechos femeninos, aunque en ocasiones haga eco de los discursos sociales que definen a las mujeres como asociadas al discurso de la domesticidad y del ángel del hogar (ver Montero, "Textos en contexto").

Durante la década del veinte, el Partido Cívico Femenino elaboró un discurso de defensa de los derechos de las mujeres, a partir de la educación de las conciencias femeninas y difundiendo la cultura cívica, cuestión que realizó a través de actividades públicas y con su revista Acción Femenina. Además, fue una organización autónoma de cualquier grupo político o religioso, definiéndose laica, democrática e independiente. A la vez, el partido unió las reivindicaciones jurídicas y políticas con reivindicaciones que eran específicamente femeninas, con lo que dieron un paso adelante en el feminismo moderno (Kirkwood 152). Julieta Kirkwood aplaude del Partido Cívico Femenino su postura de unidad de todos los grupos feministas y de mujeres; sin embargo, se plantea más crítica frente a la justificación de ésta, que sería la defensa de los derechos de las mujeres, pero en tanto cumplen la labor de la maternidad. La unidad de los distintos grupos, ya fueran católicos, de izquierda o de derecha, se entiende por las precarias condiciones en las que estaban viviendo las mujeres. Sin embargo, la autora hace notar que el Partido Cívico Femenino era contradictorio en sus planteamientos, ya que, por una parte reconocía la necesidad de que las mujeres salieran del sometimiento, para lo que había que postular un nuevo orden, sin embargo, por no querer echarse al mundo encima, terminó por postergar la revolución sexual feminista. Esta contradicción se acompañó con otra, referida a la necesidad de equilibrar la exigencia de desarrollo e independencia de las mujeres con la de afecto, lo que el partido resolvió concentrándose en la familia (Kirkwood 155-156). A partir del análisis realizado en trabajos anteriores (Montero, "Contrapunto"), se hace necesario revalorar el discurso feminista del Partido Cívico Femenino, en su completa dimensión, ya que, si bien está cruzado por tensiones, éste responde al espacio público en el que se expresó. Esto significa que, para legitimarse como un hablante más, tuvo que realizar una serie de negociaciones con los poderes institucionales que delimitaban la acción de 
las mujeres. De tal forma, el discurso feminista que se expresa en Acción Femenina resignificó los roles tradicionales asignados a las mujeres, como la domesticidad y maternidad, para defender derechos políticos y sociales (Montero, "Feminist Journals").

El Movimiento Pro Emancipación de la Mujer Chilena, y La Mujer NUEVA

El MEMCH se formó en mayo de 1935, a partir de la reunión entre Elena Caffarena y Marta Vergara, quienes junto a ocho mujeres más formaron la dirección de este nuevo movimiento. Tenían la firme idea de organizarse para luchar por los derechos y bienestar de las mujeres, declarándose decididamente feministas e independientes de todo partido político. Según ellas, era la única forma de hacer efectiva la demanda por los derechos femeninos, partiendo de la base de que las propias mujeres asumieran que la reivindicación de sus derechos correspondía a demandas específicas de ellas. Afirmaban, además, que los varones, independiente de su posición política, no estaban interesados en la emancipación femenina, y aún más, muchos creían que era una cuestión perjudicial para la sociedad. A la vez, criticaban la idea de la izquierda de que la liberación de las mujeres sería resultado de la liberación social mayor, pues eso nada más escondía la indiferencia de los hombres de izquierda ante la sujeción de las mujeres (Antezana-Pernet 51-62).

El feminismo del мемсн fue radical para su época. Su discurso incluyó una lectura de género para cada uno de los problemas políticos y sociales de la década, planteando las consecuencias que tenían ellos sobre las mujeres, y proponiendo soluciones que las incluyeran. De tal forma, el MEMCH realizó marchas, propuso leyes, discutió con parlamentarios, entre muchas otras actividades. Su radicalidad se puede observar en cuestiones como en la elección de su nombre que incluía la palabra emancipación, la que, asociada a mujeres, era toda una provocación para la época. Otro tema fue la resignificación de la maternidad, por ejemplo, la que no negaron como una cuestión propiamente femenina, pero cuyo ejercicio defendieron en las mejores condiciones. Esto significó la lucha por los descansos pre y post natal para las obreras, aborto cuando las condiciones sociales impedían una buena crianza para los hijos, entre otras cuestiones. 
Terminaron por convertirse en la organización más grande de su tipo, con presencia en todo el país, estableciendo una directiva en Santiago y una serie de comités provinciales y de barrios. Como principios de base de la organización estaban el feminismo y la democracia, lo que le otorgó una orgánica particular. Por ejemplo, en la dirección del мEMCH las decisiones eran tomadas por unanimidad, y se debía argumentar cada intervención. A nivel nacional, cada comité decidía su propia forma de organización, pero siguiendo las directrices resultantes de los congresos nacionales, que fue la fórmula para consolidar la organización. Cada congreso nacional fue una forma de educación política, formación ideológica y disciplina organizacional; por su parte, la elaboración de la revista La Mujer Nueva fue otra estrategia para establecer unidad y entregar las líneas de acción del movimiento.

El MEMCH estaba compuesto por mujeres de diversas clases sociales; sin embargo, en Santiago y en la dirección del movimiento predominaban las mujeres de clase media educada, y profesionales, muchas de ellas empleadas públicas. Sus actividades laborales las motivaban a participar del movimiento, ya que eran testigos de las condiciones sociales deplorables de la población que atendían. Por otra parte, la gran mayoría de las mujeres que formaban parte de los comités de barrio eran mujeres obreras o empleadas domésticas, que se acercaron al MEMCH porque vivían en carne propia las injusticias como trabajadoras y mujeres. Si bien en el discurso el мемсн hablaba de igualdad entre las integrantes, en la práctica se establecían relaciones jerárquicas, donde las mujeres profesionales tenían la función de educar a las obreras. Sin embargo, esta política tenía una función estratégica, ya que si la organización quería crecer a lo largo del país y de forma interclasista, se buscaba que mujeres profesionales asumieran la cabeza de un grupo, de forma que fuera atractivo para otras mujeres de clase media; en un país donde se establecían diferencias sociales tajantes, era difícil que en provincias las mujeres de clase media quisieran participar en condiciones de igualdad con mujeres obreras o empleadas domésticas (Antezana-Pernet 123).

Formar parte del мемсн no dejaba de ser una acción de valentía, ya que era una organización que era mirada con sospecha, tanto por ser declaradamente de izquierda como por ser feminista. Este tipo de organización estaba condenado por la Iglesia, cuestión no menor en Chile, donde la gran mayoría se declaraba practicante del catolicismo, sobre todo 
las mujeres. Muchas de las mujeres que participaron del MEMCH nunca terminaron por inscribirse formalmente; y muchas de quienes lo hicieron, acudían a las reuniones a escondidas de sus familias (Antezana-Pernet 140).

EL DISCURSO FEMINISTA EN DIÁLOGO CON LOS DISCURSOS SOCIALES. LOS PROBLEMAS DE SALUD: MORTALIDAD, MATERNIDAD Y PUERICULTURA

La salud se transformó en uno de los temas de discusión fundamentales de la década del treinta. Para la época, existía la percepción generalizada de que Chile poseía una sociedad enferma, dadas las altas tasas de mortalidad, tanto adulta como infantil. Los distintos grupos sociales y políticos compartían este diagnóstico, aunque diferían en el análisis de las causas. Por otra parte, el discurso de la eugenesia y el higienismo se impusieron, llegando a hegemonizar las políticas públicas relativas a la salud, e interviniendo no solo en ese ámbito específico, sino que extendiendo su influencia a la educación y el urbanismo, entre otros aspectos. La influencia de estos discursos se relaciona con el proceso de consolidación de los Estados nacionales a principios del siglo xx, el que implicó un crecimiento del aparato estatal, unido a un discurso que contribuyera a la identificación nacional de la población. En términos institucionales, esto significó una acción decidida del Estado por controlar y planificar todos los aspectos relativos a la vida social: desde la construcción de las ciudades, hasta los hechos vitales de las personas. En este proceso, el higienismo y la eugenesia fueron los discursos hegemónicos que determinaron la inauguración de la biopolítica en América Latina. El ejercicio del biopoder significó el control de los cuerpos que formaban parte de la nación de acuerdo con los parámetros definidos por el Estado, lo que equivalía a manejar los movimientos de las personas -su salud, sexualidad, sus intereses, etc.- a partir de la conformación de una nueva institucionalidad. De tal forma, se aplicó en el urbanismo la conformación de instituciones de salud, educación, vivienda, comercio, etc. (Outtes 7-29).

Higienismo y eugenesia, alzados como discursos hegemónicos, se relacionaron con los demás discursos sociales y se pueden reconocer en los diversos tipos de textos, a través de relaciones de acuerdo y/o disputa; que para el caso de su relación con el discurso feminista, resultaron ser elementos que, dependiendo del tema en discusión, el feminismo recogió 
o contradijo. La relación del feminismo con estos discursos hegemónicos (el higienismo y la eugenesia) le imprimió características específicas, que lo hacen un discurso feminista particular.

Ahora bien, la presencia del discurso higienista en América Latina se relaciona con el desarrollo de éste desde su origen como ciencia médica en Europa hacia el siglo XVII, instalándose en nuestra región a partir del siglo xix. Esta ciencia relacionó las enfermedades, el entorno urbano, las condiciones de vida y de trabajo de las personas. Buscó respuesta a las altas tasas de mortalidad, que se veían aumentadas en la población más pobre, que vivía en condiciones de hacinamiento, desnutrición y en ambientes laborales peligrosos (Alcaide).

El desarrollo del higienismo se planteó intervenir en los programas de salud pública para mejorar el perfil sanitario urbano, lo que en América Latina significaba acercarse a los modelos europeos y estadounidenses. El objetivo final era conseguir, a largo plazo, el descenso de la mortalidad y la morbilidad de la población, interviniendo y controlando a la población a través de una gran cantidad de variables tanto públicas como privadas: "la higiene social se proponía rodear a la población con un ambiente limpio, enseñarle los fundamentos del aseo personal y modificar sus hábitos, con miras a prevenir la enfermedad y asegurar la salud de las generaciones venideras" (Lavrin 138).

La higiene abarcó todos los campos de la vida humana: tanto los que se relacionaban con el medio ambiente -como la atmósfera, el clima, las habitaciones, alimentos, bebidas-, y los relacionados con el ser humano en sí, como el funcionamiento fisiológico, el temperamento, las pasiones humanas, la idiosincrasia, la herencia. De tal forma, el higienismo no solo se ocupó de las enfermedades del cuerpo individual, sino que se preocupó de la sociedad en su conjunto, interviniendo en ámbitos comerciales, para el control de mataderos, mercados y comercios en general; industriales, para verificar el funcionamiento de manufacturas e industrias; eclesiásticos en relación con los cementerios; y civiles, para la supervisión de las propiedades privadas y públicas. Estas últimas, en función de la construcción de la infraestructura urbana necesaria para mantener la salud de la población: vías urbanas, viviendas, evacuación de aguas, edificios públicos, entre otros. Además, se preocupó del control de los individuos en relación con su comportamiento moral, buscando la eliminación de la prostitución, la vagancia, el alcoholismo (Alcaide). 
Por su parte, la eugenesia fue una ciencia que surgió a fines del siglo XIX y que tenía como objetivo lo que consideraba como mejoramiento de la raza a través de la intervención en la selección natural de la reproducción, proponiendo una selección racional. Su idea era controlar genéticamente las características de la población de forma que se reprodujeran solo los sujetos definidos como aptos para el progreso. A la vez, buscaba evitar la degeneración de la raza causada por las enfermedades venéreas y los males sociales, por lo que el Estado vio en esta ciencia una aliada para estimular la reproducción selectiva. De estas acciones se derivó el desarrollo de la eugenesia positiva, que se planteaba seleccionar a los que consideraba aptos para una reproducción saludable; y la eugenesia negativa, que impedía la reproducción de débiles y enfermos. Sin embargo, no se definía lo que se entendía por características positivas o negativas, solamente se establecía que las enfermedades y sus efectos eran cuestiones que se quería evitar, integrando en ellas, sin distinguirlas, la locura, la prostitución, la delincuencia y el retardo mental. La promesa final de la eugenesia era extender la esperanza de vida, traducida en reproducir las mejores condiciones físicas y psíquicas (Lavrin 150).

En Chile, la eugenesia cobró fuerza entre 1924 y 1938, estableciendo un discurso que definía las condiciones deseables para los habitantes del país, que incluía características biológicas, físicas y morales superiores, para generar patrones sociales homogéneos y sanos (Labarca 90). La eugenesia se vistió con traje de ciencia salvadora de la población, ya que se presentaba como solución para alcanzar una mejor calidad de vida, impidiendo la transmisión de características hereditarias negativas y sus consecuencias en la generación de enfermedades mentales, delincuencia, alcoholismo y vagancia. Los profesionales vieron en la eugenesia la clave para aumentar el crecimiento demográfico y eliminar los problemas de salud. Para ello se relacionaron con el higienismo, centrándose en el control de la sexualidad, a través de reformas sociales y de la salud pública y privada (Lavrin 139). Otro ejemplo de la influencia de la eugenesia en el Estado chileno fue el decreto ley sobre "Defensa de la Raza" en 1925, que explicitaba como labor del gobierno la lucha contra las enfermedades y las costumbres que causaban degeneración, estableciendo medidas de control de ellas. Frente a este discurso, muchos reformadores sociales de ascendencia liberal, socialista o anarquista, hicieron ver los peligros de esta ciencia, en tanto que pasaba por alto los derechos de las personas, y no consideraba factores explicativos de los males sociales fuera del sujeto en sí. 
En relación con el ideal de la feminidad, la higiene social y la eugenesia recogieron las ideas tradicionales en torno al rol de las mujeres en la sociedad, y legitimaron, de cierta forma, la participación femenina en la reforma social. Bajo esta perspectiva, las características de las mujeres eran funcionales a la reproducción y el cuidado de la población. De la misma forma, la eugenesia vinculaba la educación sexual con el cuidado de la población y la salud infantil, por lo que las mujeres eran equiparables con los varones en relación con la reproducción (Lavrin 140). El discurso feminista hizo eco de estos discursos, lo que representa un matiz en el feminismo de estas organizaciones chilenas.

Uno de los indicadores de la crisis que se vivía en la década, y que repercutió en la salud de la población, fue la alta tasa de mortalidad. Ahora bien, las estadísticas no eran exclusivas de Chile, pues los países latinoamericanos en general mostraban alta mortalidad desde principios del siglo xx, alcanzando, en promedio, 254 por cada mil (Anuario estadístico 4), siendo la tuberculosis la principal causa de muerte. Lo que distinguió a Chile de otros países latinoamericanos fue que, avanzado el siglo $\mathrm{xx}, \mathrm{y}$ específicamente en la década del treinta, la mortalidad no bajaba y, más aún, la infantil era la más alta del mundo (ver Allende 80-81) ${ }^{3}$.

Los distintos discursos sociales que denunciaron las altas tasas de mortalidad se acompañaban de un diagnóstico catastrófico de las condiciones de vida de la población, especialmente de los pobres (problemas de vivienda, red de servicios básicos insuficiente, malnutrición, entre otras cuestiones), los que se confabulaban y hacían de la salud un problema público. De esa forma lo entendieron las mujeres feministas, tanto las del Partido Cívico Feminista, como las de MEMCH. Ambas subrayaron lo social de las causas en la mortalidad de la población, por ejemplo el PCF declaró:

Causas de mortalidad tomadas por biológicas, no son sino CAUSAS SOCIALES. La influencia de estas causas sociales, según que el infante nazca y se desarrolle en una u otra condición, se revela todavía de un modo más decisivo por la influencia de la situación económica de los padres. Hay una mortalidad mucho

\footnotetext{
Nos cabe la duda de la existencia de estadísticas completas para todos los países, dada la época.
} 
mayor en los infantes de las clases proletarias y poco instruidas (Keltz 40).

Como diferencia, el MEMCH incluyó una crítica política al momento de denunciar las malas condiciones de vida de la población en Chile, en este caso al gobierno de Alessandri y sus autoridades. Este gobierno de derecha ganó las elecciones de 1932 y desplegó un discurso en el que atacó a quienes consideraba una amenaza para el orden social, como socialistas y las organizaciones populares asociadas al Partido Comunista (Correa et al.).

Según la última Sinopsis de la Dirección General de Estadística, en 1932 fallecieron 235 menores de un año por cada mil niños nacidos vivos... el porcentaje de niños muertos el último año ha aumentado a 262.

El nivel extraordinariamente alto de mortalidad por debilidad congénita y vicios de conformación demuestra que la alimentación y cuidado de la mujer embarazada es deficiente y que así hoy por hoy nuestro primer deber es proteger a la madre trabajadora, a la madre del pueblo chileno.

¿A pesar de todo esto, la señora regidora por Santiago, doña Elena Doll de Díaz, ha dicho que estamos en Jauja! ("iLa resistencia de la raza...!" 1).

La denuncia cobra fuerza al terminar la descripción, ironizando las palabras de las autoridades de gobierno, quienes en el contexto de asentar su estabilidad política, declaraban estar al mando de un gobierno de unidad y desarrollo para Chile.

Frente a la situación de crisis social y sanitaria, el discurso eugenésico alzó la voz de alerta, a través de políticos y profesionales estatales. El anuncio era que la patria se debilitaba y se detenía el desarrollo potencial del país; y de la misma forma lo entendieron las feministas del PCF, quienes reforzaron la idea de que el progreso se aseguraba aumentando el número de su población. "Un país es tanto más próspero cuanto mayor es la densidad de población. La mortalidad infantil constituye una sangría abierta que merma la densidad de población y el engrandecimiento del Estado" (Folch de Rosés 14-15).

De la misma forma, el discurso eugenésico planteaba que la atención del Estado debía enfocarse en los obreros, ya que era el grupo social que más contribuía a la riqueza de la sociedad. En este punto, el discurso feminista 
se tensa, ya que apoyó la idea de considerar a este grupo como "reserva de vida", criticando las prácticas sexuales de control de la natalidad, a pesar de que a partir de otros problemas sociales defienden la necesidad de anticoncepción y aborto:

La disminución de la frecuencia de los nacimientos, como la expresión de una limitación voluntaria de la concepción, indica un elemento de degeneración. Por este motivo, parece que esta "racionalización" de la vida sexual, ha penetrado en las clases populares, que constituyen la reserva de hombres de la nación (Keltz 40).

A partir de la idea de concebir a la clase obrera como la principal gestora de vida, el discurso eugenésico cosificó a los obreros, calificándolos como sujetos pasivos, lo que se tradujo en que se los definió como objetos de políticas públicas. Según la eugenesia, era la sociedad en su conjunto quien debía procurar el bienestar de todos, y generar las condiciones necesarias para que se desarrolle la vida en condiciones óptimas y el consecuente nacimiento de una generación saludable (Lavrin 166).

La raza influye sobre la mortalidad infantil, pero quizá gran parte de lo que a ella se atribuye se deba a las diversas condiciones económicas y morales de los distintos pueblos. No obstante parece que existe una superioridad biológica en ciertas razas que da a los niños una mayor resistencia contra las enfermedades. Tal ocurre entre los rusos.

Pero la pobreza de las clases proletarias, la extrema indigencia de los parados jles aleja tantas veces del establo donde hay una buena leche para los niños! Y luego en las habitaciones pequeñas y superpobladas, los rigores el verano se extreman, los gérmenes de la leche se multiplican exageradamente (...) el niño pequeño se pone en contacto con objetos contaminados con adultos enfermos (Poch y Gascón 42-44).

En este texto, si bien Acción Femenina recoge el elemento eugenésico que tiene una interpretación discriminatoria frente a la genética nacional, finalmente pone el acento en los problemas sociales, como origen de la debilidad de los cuerpos chilenos.

Por su parte, el discurso higienista planteaba la necesidad de comprometer al Estado para mejorar la salud pública a través de la 
prevención. La higiene social presuponía que la población era ignorante, frente a lo cual planteó la necesidad de establecer estrategias de limpieza ambiental y enseñanza del valor del aseo personal, haciéndolo un hábito (Lavrin 138). El siguiente texto, publicado en Acción Femenina, recoge estos elementos del higienismo y los transforma en una oda al cambio social higiénico, reforzando las ideas discriminatorias que éste suponía:

Reúne todos los conceptos para enriquecer la vida, y seleccionar a los padres de la raza, y difundir una sangre pura en el niño que vigilaremos aún antes de nacer.

Cuando decimos salud pública y deseamos la salud pública y tenemos el alma y el cuerpo limpios, sentimos el poder de esta pureza que llevamos para sostener dignamente el alma.

$\mathrm{Al}$ decir salud pública nos imaginamos la alta cultura del pueblo amante del agua, el interés de los higienistas por embellecer la ciudad e instruir a los hombres incapaces.

El cuerpo, el templo del espíritu, debe ser un vaso límpido forjador de alegrías y de fuerzas que nos encaminan hacia el ideal.

$\mathrm{Y}$, entonces pensaremos en plasmar el alma del niño y de conducirle por el camino.

Porque la suciedad del cuerpo y del ambiente empañan el crisol del alma y se pervierte la pureza del Espíritu en un cuerpo degradado en cuyas venas corre una sangre infecciosa.

La ciudad ideal sería de los bellos jardines y de limpias aguas que generosas se dieran al desposeído; al paria social, es decir a los más pobres; a los que nos les alcanzan sus medios, para liberarse del mal, el microbio y la infección y el desmoronamiento total de sus energías.

La Ciudad Luz sería aquella que levantara el hogar del pobre. Que vigilara el ambiente de la madre y el niño.

Que sostuviera la Ley del Hogar en los matrimonios obreros bajo la vigilancia celosa del bien colectivo (de Sotomayor 32 y 36 ).

Este texto nos muestra una tensión entre el discurso higienista y el feminista. Por una parte, recoge sin crítica los principios del higienismo, al que incluso considera un ideal -que es como se titula al artículo-, cuestión que se refuerza con el tono utilizado y las cualidades de la ciudad ideal. De la misma forma, equipara la salud moral con la salud física, lo que se repite en múltiples artículos de la revista. La consecución del ideal implica una regulación o la preeminencia de la norma. Sin embargo, el discurso 
feminista hace notar que la infección, finalmente, es de origen social y no sería esencial a la población más pobre.

Los discursos sociales que abordaron los temas de salud se enfocaron en la mortalidad infantil, la tuberculosis, el cuidado de las mujeres embarazadas y la puericultura. Al ser definidos como problemas, el discurso estatal los culpó del debilitamiento de la nación, y los elevó a la categoría de preocupación nacional. De tal forma, se planificaron políticas de salud pública que buscaban ser eficaces en la protección de los niños, el cuidado de las embarazadas, de forma que crecieran ciudadanos fuertes y se terminara con las bacterias que infectaban al país.

Estas ideas llevaron a definir un discurso estatal que ponía su acento en las madres. Ellas se transformaron en pieza clave de las políticas públicas, ya que dado su rol tradicional de madres y cuidadoras de vida se plantearon medidas para protegerlas; sin embargo esa protección suponía que ellas eran las responsables finales de la salud de la nación. Y de esa forma lo entendieron en Acción Femenina al afirmar que:

Una de las causas principales de la mortalidad infantil es la ignorancia, por una parte, en lo relativo a la alimentación; el ABANDONO en buscar el consejo del médico en el principio de una enfermedad; la MISERIA y en general desconocer por completo el cuidado que requiere un niño (Folch de Rosés 14-15).

La higiene social buscó educar a las mujeres de la creciente clase media como enfermeras y asistentes sociales para ir en ayuda de los pobres enfermos, y enseñar a las mujeres en general a cumplir un mejor papel como madres (Lavrin 140). Este rol definido por los discursos sociales tradicionales en torno a las mujeres, ya conocido por ellas, fue recogido como una labor social. Frente a ello, utilizaron los elementos del higienismo que planteaban lo científico como justificación de la acción de las mujeres en el espacio social: "Las mujeres somos el eje del mundo debido a la maternidad. Es enteramente necesario, entonces, que nos preocupemos entonces seriamente, de robustecer en forma científica, tanto nuestro espíritu como nuestro cuerpo" (Villarroel 10).

En relación con la maternidad, el discurso higienista se centró en las madres obreras, y estableció una relación entre madre, hijo y condiciones laborales que tuvo características reaccionarias, llegando a criticar el trabajo femenino fuera del hogar. Sin embargo, para las mujeres feministas esta 
situación era una expresión más de la desigualdad social que vivían las mujeres: no solo eran pobres, sino que además, en tanto madres, debían cargar con los hijos y su cuidado, por lo que urgía mejorar las condiciones de trabajo de las mujeres.

La madre misma girando en el círculo de su pobreza que la arrastra, a veces a trabajos inadecuados, no puede atender al chiquillo y todavía no hay salas cuna ni guarderías suficiente para todos.

La madre abandonada se encuentra en condiciones de vida social y económica muy difíciles que repercuten sobre el niño. El niño, que supone casi siempre una carga para la madre, tiene que soportar la privación de alimentos e higiene (Poch y Gascón 42-44).

Se puede observar al feminismo cuestionando al discurso higienista, que definía como ignorantes a las madres pobres y las responsabilizaba por la mortalidad infantil, ya que, dadas las condiciones económicas, aunque las mujeres supieran las medidas higiénicas necesarias para evitar enfermedades, sus condiciones materiales les impedían alejar a sus guaguas de la humedad, o no tenían recursos para mejorar su dieta.

Por otra parte, dada la desregulación laboral, persistía la imagen de mujeres que al otro día de parir continuaban con su labor en las fábricas (Zárate, "Madres obreras: identidad social" 64); para qué hablar de la ausencia de cuidados prenatales o contar con medios para controlar complicaciones del parto, situación consolidada por la falta de hospitales. En la siguiente imagen (figura 1) se puede observar la utilización política de la denuncia: las mujeres constatan la falta de protección a las madres, y llaman a votar por su candidato de izquierda, Pedro Aguirre Cerda, quien al apoyarlas como grupo político se haría cargo de las injusticias sociales en las que vivían las mujeres pobres: 


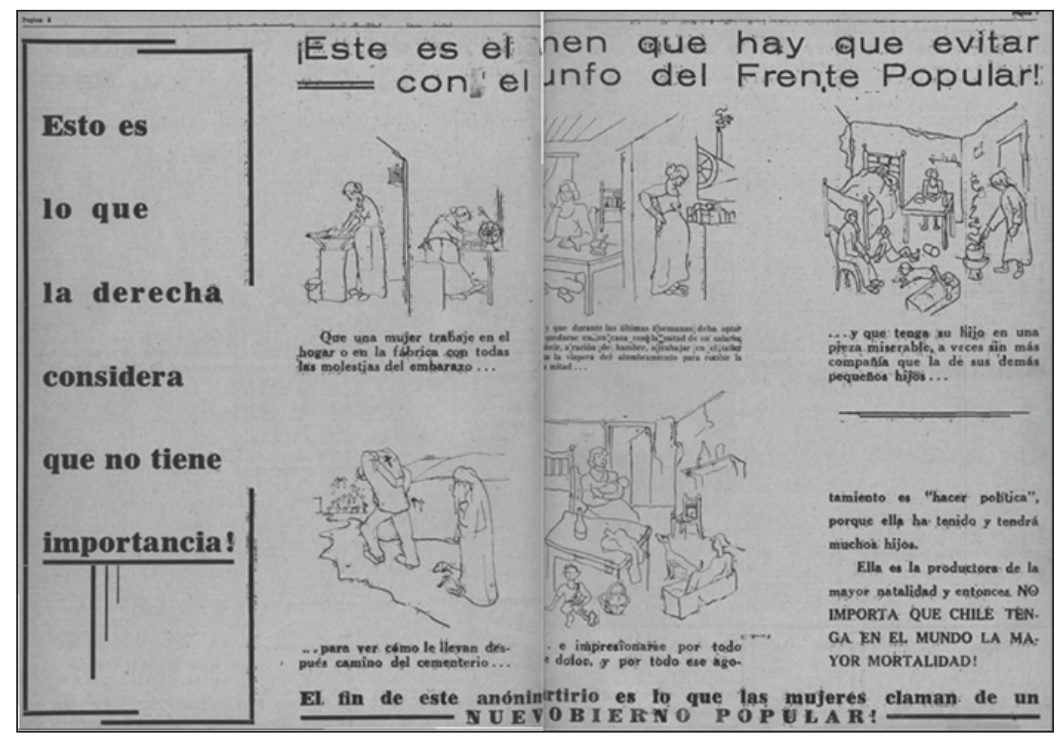

En 1925, la promulgación del Código del Trabajo consideró un permiso laboral de sesenta días para las trabajadoras embarazadas, el que sin embargo no se cumplía, o los empleadores buscaban tretas para no cumplirlo. En 1929, el Gobierno de Ibáñez, recogiendo los principios higienistas, elaboró planes con financiamiento fiscal y municipal para apoyar a las obreras embarazadas hasta cuatro meses después del parto, con el objetivo de conservar la salud del pueblo, por lo que se consideró la gratuidad del servicio para los más pobres. El mismo espíritu se trató de recoger en el Código del Trabajo de 1931, que proponía la protección de madres e hijos, y que se trató de mejorar aún más en el Código de 1935, pero que finalmente quedaban en letra muerta.

El patrón está obligado a proporcionar a la mujer embarazada dos semanas de permiso antes de dar a luz y dos semanas después, con salario íntegro; pero el patrón a penas advierte en su fábrica una obrera en tal estado, la lanza a la calle sin mayores explicaciones, derivándose de este acto criminal toda serie de funestas consecuencias para la obrera (Román).

Independientemente de la idea higienista de protección, la falta de 
cumplimiento de la ley alimentó la denuncia en el discurso feminista del MEMCH, otorgándole fuerza a la demanda por los derechos de las mujeres madres y haciendo de la protección una cuestión feminista. De hecho, la imagen que se utiliza para llamar al apoyo de Pedro Aguirre Cerda, corresponde a 1938, lo que demuestra el incumplimiento de las leyes de protección elaboradas hasta ese momento. A pesar de ello, se implementaron programas específicos, como el Primer Plan Trienal de Defensa del Niño, que aseguraba alimentación para los lactantes, y el año 1938 se extendió a las madres; paralelamente, se inauguró una central de pasteurización de leche y se implementó en el mismo año 1935 un programa para su distribución entre las mujeres embarazadas (Zárate, "Madres obreras y el Estado" 133). El PCF defendió los derechos de las madres y los niños a tener una buena alimentación, utilizando argumentos del discurso eugenista en torno a la necesidad de fortalecer la raza: "La gravedad que plantea este ínfimo consumo de leche no la constituye solo en que nuestro pueblo se alimente en condiciones deficientes, sino que a su trascendencia para el futuro de nuestra raza" ("Sin comentario" 3).

Los cambios legislativos se tradujeron en la formación de consultorios "Madre y Niño" en las principales ciudades del país, donde se atendía a las mujeres embarazadas y a sus hijos hasta los ocho meses. En ellos, se proveía de atención médica, remedios y alimentos como leche, pasando de ser una institución de beneficencia a la Caja del Seguro Obrero en 1942. Por otra parte, también se consideró el pago de subsidios en dinero y alimentos como estímulo para asegurar el control postparto y vigilar la lactancia materna; sin embargo, esta medida fue criticada, porque se dudaba del uso del dinero que pudieran hacer las mujeres de él. Fue igualmente controversial la propuesta de 1939 de Salvador Allende, para desincentivar el aborto y estímulo para el control prenatal, de entregar un bono en dinero en efectivo a las mujeres embarazadas. Esta propuesta se ha reconocido como el antecedente de la asignación familiar (Zárate, "Madres obreras y el Estado" 134).

La legislación de la década del treinta incluía la protección a las madres en tanto eran las reproductoras de la sociedad (Lavrin 140); sin embargo, detrás de ella estaba la influencia de discursos extranjeros, como el mutualismo obrero, el feminismo europeo, el reformismo sanitario y los seguros sociales (Zárate, "Madres obreras y el Estado" 131). En este sentido, podemos considerar un triunfo del feminismo la consideración de la maternidad como un elemento a proteger por el Estado. Sin embargo, 
dado el incumplimiento de la letra, el feminismo en Chile continuó demandando protección materna. Por ejemplo, podemos ver en el discurso del MEMCH la defensa de una maternidad digna, a partir de la denuncia de las malas condiciones de vida de las mujeres pobres. De la misma forma, se adelanta una postura crítica a la maternidad obligatoria, defendiendo el aborto como medio de control de la mortalidad infantil:

Así, mientras no llegan días mejores en que se le pueda proporcionar a la madre obrera todos los cuidados dignos de la majestad de su caso, mientras no se le asegure el pan, el techo para el abrigo para ella y para su hijo, mientras la maternidad constituya una maldición para la mujer y para la sociedad, un desfile de pequeñas criaturas desde el vientre materno al cementerio, nosotras vamos a propiciar el aborto legal, y vamos a secundar ampliamente la labor de los médicos en tal sentido (M. V. 1).

Una vez producido el parto, las políticas públicas se centraban en la protección de los niños, como símbolos de la nueva nación, libre de enfermedades y con un futuro de desarrollo (Zárate, "Madres obreras y el Estado"). Por lo tanto, la puericultura se transformó en una ciencia fundamental, que debía ser un puntal en las políticas de salud pública. En ella, las mujeres cobraban especial importancia, ya que representaban el deber ser del ideal femenino; de tal forma, el PCF replicó los argumentos higienistas responsabilizando a las mujeres por el éxito o fracaso de la crianza de los hijos, con lo que su discurso feminista se vuelve a tensar. De la misma forma, se suman al discurso cientificista que elevó la crianza a categoría de ciencia, enterrando los conocimientos tradicionales:

No nos cabe duda que en esta mortalidad, entre otros factores de índole diversa, influye grandemente la ignorancia de las madres en todo lo que se refiere a higiene y cuidados del embarazo y a la atención del niño que ha nacido.

Consideramos que la enseñanza de estos conocimientos debe ser hecha por personas que hayan cursado estudios especiales al respecto. Creemos que nos expondríamos a un fracaso si pretendiéramos que estas lecciones de Puericultura fueran dadas por profesoras que sigan enseñando a sus alumnas los mismos errores y prejuicios que ellas has aprendido en sus hogares y que van transmitiendo de generación en generación (Araya 4-6). 
La preocupación por el desarrollo de la puericultura por parte del discurso estatal se tradujo en la publicación de libros, la organización de congresos internacionales y la integración como asignatura en las escuelas básicas y en la formación de las profesionales de la salud y el servicio público. En todos ellos se reafirmaba que la puericultura era un elemento fundamental de la higiene social, y se insistía en que las mujeres eran sus sacerdotisas, aunque los elaboradores de las políticas públicas eran los varones. De la misma forma, la necesidad de extender la enseñanza de la puericultura ya no era vista como un asunto relativo a la caridad, como era en los años veinte, sino que se planteaba como un servicio a la nación. Ejemplo de ello fue la incorporación, a partir de los treinta, de la puericultura como asignatura en las Escuelas Normales 1 y 2 de Santiago, además de la organización de la Liga de Madrecitas, que seguía el ideal norteamericano dentro del modelo de popularización de la salud pública; y de tal modo lo reforzó el feminismo de Acción Femenina:

El conocimiento del niño y de la higiene infantil, es el porvenir de nuestra raza. A las mujeres, ya desde niñas por ser ellas las destinadas a ser en los venideros días, que seguirán a su infancia y pubertad, las madres, que deberán cuidar de la crianza de sus hijos, se les debiera enseñar conocimientos que las hicieran aptas para ejercer su misión sin cometer grandes errores, que aún hoy se observan, incluso en las familias de buena condición social.

Una madre, para criar bien a sus hijos, debe estar imbuida de los dos principios en que puede resumirse la puericultura moderna: higiene moral e higiene alimenticia (Folch 24-25).

Paralelamente, la Dirección de Educación Sanitaria organizó conferencias con el objetivo de estimular a los padres para que apoyaran la iniciativa del gobierno de realizar exámenes médicos a los niños; y el apoyo a la enseñanza de la puericultura a las niñas con hermanos menores, para alentarlas a convertirse en ayudante de sus madres, y con la perspectiva de su futura maternidad.

Las costumbres han cambiado tanto en estos últimos tiempos y la Ciencia ha realizado tantos progresos, derrumbando aquí y edificando allá, que la verdad de ayer no es la de hoy. Nuestros pequeños, al abrir los ojos a la luz comienzan a ser fajados de distinta manera a como lo fuimos nosotros y, por tanto, para nada nos sirven esos legajos de experiencia, que llenos de buena fe, nos brindan nuestros mayores ("Puericultura, ciencia moderna" 22). 


\section{CONCLUSIÓN}

Se ha analizado el discurso feminista y su relación dialógica con otros discursos sociales, como el higienismo, la eugenesia y el discurso del Estado, considerando debates en torno a problemas de salud en la década del treinta en Chile. El discurso ha sido el eje articulador del análisis, definido como una producción social que expresa la subjetividad de quien lo emite. El discurso feminista es pensado como contradiscurso, en la medida en que cuestiona el discurso hegemónico que ha establecido codificaciones jerárquicas que asignan características y funciones sociales y culturales a lo femenino y masculino. El contradiscurso se ha conformado a través del diálogo con el discurso hegemónico, mediante una relación de contestación o contradicción. Sin embargo, el discurso feminista, en tanto discurso social, también se compone del diálogo con otros discursos sociales, con los que establece acuerdo o contradicciones, entre otras posibilidades. Dependiendo del tema al que se refiera, el discurso feminista se tensa al reproducir ideas del discurso hegemónico que pueden ser entendidas como contradicciones.

Si los discursos sociales están cubiertos por su entorno, es decir, por las condiciones de posibilidad que les dan sentido, a la vez que dan cuenta de los procesos significantes de la sociedad, entonces, la relación del discurso feminista con los otros discursos sociales muestra cómo se construyó el feminismo en Chile en los años treinta. Esta construcción se dio, por una parte, a partir de elementos que eran significativos para las mujeres en su relación con el conjunto de la sociedad, surgidos del análisis de género que cuestiona la condición de subordinación de las mujeres, y que le hace sentido al propio colectivo feminista. Y, por otra parte, con los elementos que integran otros discursos sociales, con los que se relaciona de distinta forma: acuerdo, desacuerdo, reacción, entre otros. Dependiendo del tipo de elemento que recoja y la relación que establezca con él, el discurso feminista adquirió ciertas características.

De los discursos sociales presentes en la década del treinta, el higienismo y la eugenesia cobraron un peso muy importante, y pueden ser considerados discursos hegemónicos. Estos fueron emitidos por los profesionales que formaron parte del Estado desde el inicio del siglo xx, reproducidos por las instituciones estatales y por los distintos discursos sociales. Tanto el higienismo como la eugenesia pueden ser reconocidos 
recorriendo distintos textos en los que establecen relaciones de acuerdo y/o disputa. En su relación con el discurso feminista, dependiendo del tema que se tratara, el feminismo recogió o se contrapuso a sus principios. De tal forma, una idea eugenésica o higienista era recogida y replicada por el feminismo sin cuestionamiento en un tema determinado, o absolutamente rebatida en otro. Esta relación, que puede ser aparentemente contradictoria, expresa las negociaciones que debe hacer el discurso feminista de acuerdo al contexto en el que se desarrolla.

Los discursos feministas analizados fueron emitidos por dos organizaciones feministas que poseen diferencias en sus planteamientos, los que se manifiestan, además, en su relación dialógica con los discursos sociales, ya sea de acuerdo o disenso, dependiendo del tema. Por una parte, el Partido Cívico Femenino es una organización con mayor trayectoria, y da cuenta de un colectivo de mujeres heterogéneo, que se trasluce en el discurso feminista que plantea. Esa heterogeneidad impide unificar el discurso feminista del PCF, y finalmente se traduce en un discurso moderado e irregular en sus contenidos. Esto significa que presenta variaciones en sus posturas, tanto dentro de un mismo número, como a lo largo de la vida de la publicación.

Por su parte, el MEMCH plantea un discurso más homogéneo, dada la propia característica de la organización que desde un principio se planteó de izquierda y en defensa de los derechos de las mujeres. Además, la organización se formó en el marco de la radicalización de las posturas de derecha e izquierda, por lo que el contexto facilitaba la conformación de un discurso que no necesitaba establecer tantas negociaciones al momento de plantear posturas críticas.

En el diálogo del discurso feminista (considerado como el conjunto de los discursos de ambas organizaciones) con los otros discursos sociales, este se relacionó de distinta forma, dependiendo del tema abordado. Por ejemplo, en su relación con los discursos hegemónicos del higienismo y la eugenesia, en ocasiones los reprodujo, en otras se opuso o los cuestionó, o utilizó sus argumentos resignificándolos para plantear una postura propia. En relación con los temas de salud, el discurso feminista reprodujo elementos de la eugenesia, como el que relaciona la mortalidad adulta e infantil con la falta de desarrollo del país; o en relación con el higienismo, la idea en torno a la necesidad del ambiente limpio para conservar la salud de la población. En la medida en que muchas feministas 
de las organizaciones analizadas formaban parte del aparato del Estado, a través de instituciones de salud, educación o seguridad social, podemos entender que el discurso feminista reprodujera estos elementos animado por la necesidad de educar a la población para mejorar las condiciones de salud. Sin embargo, el feminismo agregó elementos de análisis, como la consideración de las causas sociales de la incidencia de la mortalidad en Chile, desnaturalizando la relación entre pobreza e ignorancia que realizaba la eugenesia y el higienismo.

Por otra parte, el discurso feminista establece momentos en que resignifica elementos del higienismo y la eugenesia para defender derechos de las mujeres -como cuando la eugenesia refuerza el ideal de la domesticidad como una vocación femenina para el cuidado por el otro- y justificar la acción de las mujeres frente a los problemas de salud. O la idea eugenésica de mejorar la nutrición de la población para mejorar la raza; o la necesidad de control de natalidad para evitar embarazos en condiciones materiales inadecuadas. El feminismo releyó estas ideas, imprimiéndoles una perspectiva de género para consolidar la acción de las mujeres en el espacio público a través de su acción social; o apoyar sus planteamientos relativos a que las mujeres eran las responsables de la mantención de los hijos; o la defensa del derecho a la reproducción voluntaria sin importar la condición social de la mujer. Sin embargo, frente a planteamientos eugenésicos sobre la necesidad de limitar la reproducción de las clases populares, se opuso firmemente.

El discurso feminista muestra complejidad al asumir temas que no necesariamente podrían ser catalogados como problemas políticos en general, pero que desde la perspectiva del feminismo son necesariamente cuestiones políticas que afectan a las mujeres, como la salud. La tensión surgió en su relación con los otros discursos sociales, lo que nos lleva a afirmar que el discurso feminista no puede ser considerado como una unidad discursiva homogénea o unitaria, ya que, dependiendo de la organización o colectivo de mujeres que lo emite, tiene matices. Por otra parte, el discurso feminista tomó elementos de otros discursos sociales para construir su propio discurso; en ese ejercicio, algunas veces entra en disputa con ellos y en otras ocasiones los reproduce, dando cuenta de la compleja operación que realiza el feminismo, tanto para legitimarse como discurso, como para construirse a sí mismo como contradiscurso. 
A partir de su consideración como contradiscurso, y tomando en cuenta su desarrollo dentro del entramado social, el discurso feminista adquiere matices. Ejemplo de ello ha sido revisar el feminismo chileno de la década del treinta, el que, entendiéndose a sí mismo como contradiscurso, posee contradicciones. Estas contradicciones confirman el espesor del mismo discurso feminista, en el sentido que en tanto práctica social, o movimiento político, incluye elementos para legitimarse-que le son ajenos pero que son conocidos en el espacio social-, ya sea reproduciéndolos o resignficándolos. De tal forma, independiente de su impulso teórico, el feminismo, al momento de hacerse práctica, expresa las necesidades, las características, el contexto de quienes lo encarnan, en un lugar y momento determinados.

Cobra sentido la idea de la teoría de los discursos en el sentido de que el feminismo no se construye a sí mismo desde sujetos inmaculados, sino que lo hace a partir de un grupo de sujetos con historia, en un contexto y que intentan cambiar su escenario social; y este último no solo está compuesto por el grupo que comparte sus ideas, sino que incluye a todo el resto de comunidades o grupos sociales.

\section{BiBLIOGRAFÍA}

Anuario estadístico. Santiago, 1930. Impreso.

Alcaide, Rafael. "Las publicaciones sobre higienismo en España durante el período 1736-1939. Un estudio bibliométrico". Scripta Nova: revista electrónica de geografía y ciencias sociales 50 (1999). Web.

Allende, Salvador. La realidad médico social chilena. Santiago: Ministerio de Salubridad, Previsión y Asistencia Social, 1939. Impreso.

Anderson, Bonnie y Judith Zinsser. Historia de las mujeres: una historia propia. Vol. 2. Barcelona: Crítica, 1992. Impreso.

Angenot, Marc. Interdiscursividades. De hegemonías y disidencias. Córdoba: Editorial Universidad Nacional de Córdoba, 1998. Impreso.

Antezana-Pernet, Corinne. El MeMch hizo historia. Santiago: Imprenta SEIT, 1997. Impreso. 
Araya, María. "Trabajo presentado en el último Congreso Pan Americano del Niño, en Méjico D. F.”. Acción Feminina 16 (julio y agosto 1936): 4-6. Impreso.

Berman, Marshall. Todo lo sólido se desvanece en el aire. La experiencia de la modernidad. México: Siglo xxi, 1991. Impreso.

Correa, Sofía et al. Historia del siglo XX cbileno. Santiago: Sudamericana, 2001. Impreso.

De Miguel, Ana. "Feminismos". Diez, palabras clave sobre mujer. Celia Amorós, ed. Navarra: Editorial Verbo Divino, 1995. Impreso.

De Sotomayor, Herminia. "Ideal". Acción Femenina 5 (febrero y marzo 1935): 32-36. Impreso.

Dra. KeLtz. "Sobre la mortalidad infantil”. Acción Femenina 4 (enero 1935): 40. Impreso.

Folch de Rosés, Matilde. "Mortalidad infantil". Acción Femenina 7 (julio 1935): 14-15. Impreso.

"Puericultura". Acción Femenina 8 (agosto 1935): 24-25. Impreso.

GAVIOLA, EDDA. Queremos votar en las próximas elecciones. Historia del movimiento sufragista chileno 1913-1952. Santiago: LOM, 2007. Impreso.

Grau, Olga et al. Discurso, género, poder: discursos públicos, Chile 1978-1993. Santiago: LOM, 1997. Impreso.

Kirkwood, Julieta. Ser política en Chile: los nudos de la sabiduría feminista. Santiago: Cuarto Propio, 1990. Impreso.

Labarca, Catalina. "Todo lo que usted debe saber sobre las enfermedades venéreas". Por la salud del cuerpo. Historia y políticas sanitarias en Chile. María Soledad Zárate, comp. Santiago: Ediciones Universidad Alberto Hurtado, 2008. 81-129. Impreso.

“LLa resistencia de la raza chilena disminuye día a día!” La Mujer Nueva 9 (agosto de 1936): 1. Impreso.

Lavrin, Asunción. Mujeres, feminismo y cambio social en Argentina, Chile y Uruguay, 1890-1940. Santiago: DiBAm/Centro de Investigaciones Diego Barros Arana, 2005. Impreso.

Molyneux, Maxine. Movimientos de mujeres en América Latina. Estudio teórico comparado. Madrid: Cátedra, 2003. Impreso. 
Montero, Claudia. "Contrapunto: mujeres de clase media a través de revistas. Chile y Argentina, 1920-1939”. Tesis de Magíster en Estudios Latinoamericanos. Universidad de Chile, 2005. Impreso.

"Feminist Journals in Latin America 1920-1940: A Space for the Construction of Modern Subjects". Identity, Nation and Discourse: Latin American Women Writers and Artists. Claire Taylor, ed. Cambridge: Cambridge Scholar Publishing, 2009. 17-37. Impreso.

"Textos en contexto. Discursos feministas en revistas feministas y su relación dialógica con los otros discursos sociales, Chile 19301939". Tesis de Doctorado en Estudios Latinoamericanos. Universidad de Chile, 2010. Impreso.

M. V. "El problema del aborto y la mujer obrera". La Mujer Nueva 4 [ं1936?]: 1. Impreso.

NASH, MARY. Mujeres en el mundo. Historia, retosy movimientos. Madrid: Alianza, 2004. Impreso.

OutTES, Joel. "Disciplinando a la sociedad a través de la ciudad: el origen del urbanismo en Argentina y Brasil (1894-1945)". EURE 28 (2002): 7-29. Impreso.

Poch y Gascón, Amparo. "La mortalidad infantil". Acción Femenina 12 (diciembre 1935): 42-44. Impreso.

"Puericultura, ciencia moderna". Acción Femenina 6 (mayo 1935): 22. Impreso.

Rojo, Grínor. Diez, tesis sobre la crítica. Santiago: LOM, 2001. Impreso.

Román, Eulogia. "La Mujer obrera es doblemente EXPLOTADA". La Mujer Nueva 1 (noviembre 1935). Impreso.

Sau, Victoria. "Feminismo". Diccionario ideologico feminista. Victoria Sau, ed. Barcelona: Icaria, 2000. Impreso.

"Sin comentario". Acción Femenina 16 (julio 1937): 3. Impreso.

Villarroel M, Mila. "Educación física femenina". Acción Femenina 12 (diciembre 1935): 10. Impreso.

ZÁrate, María Soledad. "Las madres obreras: identidad social y política estatal, Chile, 1930”. Revista de Historia Socialy de las Mentalidades IX. 1-2 (2005): 59-83. Impreso.

"Las madres obreras y el Estado chileno. La caja del Seguro Obligatorio, 1900-1950". Mujeres chilenas, fragmentos de una historia. Sonia Montecino, comp. Santiago: Catalonia, 2008. 129-137. Impreso. 